\title{
ROBOT EXPLORADOR PARA LA DETECCIÓN DE METALES EN ENTORNOS MINEROS
}

\section{EXPLORING ROBOT FOR METAL DETECTION IN MINING ENVIRONMENTS}

\author{
Elías Isaac Isidro Ríos', José Luis Zamora Olazábal², \\ Robert Castro Salguero ${ }^{3}$
}

RECEPCIÓN: 30 DE OCTUBRE DE 2020

ACEPTACIÓN: 12 DE NOVIEMBRE DE 2020

\section{RESUMEN}

La presente investigación presenta los pasos necesarios para el diseño y ensamblaje de un robot explorador controlado. Su utilidad inmediata es la detección de metales para las empresas dedicadas a la exploración de zonas con altas concentración de minerales metálicos. El diseño de dicho robot explorador es posible a través de la selección de los materiales y la técnica de la simulación de las piezas realizadas por el software de ingeniería SolidWorks.

Palabras clave: simulación, manufactura, aplicaciones, robot, telecontrol.

\begin{abstract}
The present investigation corresponds to the application of a controlled explorer robot for the detection of metals in companies dedicated to the exploration of areas with high concentrations of metallic minerals. The design of an explorer robot is detailed, through the selection of materials, the simulation of the parts with SolidWorks engineering software.
\end{abstract}

Keywords: simulation, manufacturing, applications, robot, remote control.

1 Estudiante de la Escuela Profesional de Ingeniería Mecatrónica de la Universidad Ricardo Palma. <elias.isidro@urp.edu.pe>

2 Estudiante de la Escuela Profesional de Ingeniería Mecatrónica dela Universidad Ricardo Palma.<jose.zamora@urp.edu.pe>

3 Ingeniero Mecánico-Electricista de la Universidad Nacional de Ingeniería. Egresado de la Maestría de Automática e Instrumentación de la Universidad Nacional de Ingeniería. Egresado de la Maestría en Docencia Universitaria y Gestión Educativa de la Universidad Alas Peruanas. Docente de las asignaturas de Mecánica del Cuerpo Rígido, Resistencia de Materiales y Cálculo de Elementos Finitos y Mecánica de Fluidos y Transferencia de Calor de la Universidad Ricardo Palma. Docente de Programación y Métodos Numéricos de la Universidad Nacional de Ingeniería. < robert.castro@urp. edu.pe> 


\section{INTRODUCCIÓN}

En estos últimos años, una de las tendencias más importantes para el crecimiento de la economía es la minería, que es una de las principales riquezas en el Perú, país que, en el mundo, ha sido catalogado como el cuarto principal yacimiento más grande. La minería, además de ser uno de los motores importantes para el crecimiento de un país, contempla muchas preocupaciones relacionadas al impacto ambiental y a la salud ocupacional que puede ocasionar con sus actividades diarias [1]. Según las estadísticas de Osinergmin, al cierre del año 2018, un 65\% de los accidentes mineros pertenecen a yacimientos de minas subterráneas, mientras que el $35 \%$ restante pertenece a las minas de tajo abierto [2].

\section{DISEÑO DEL ROBOT EXPLORADOR}

\subsection{Diseño mecánico}

Se utilizó un sistema motriz de 2 ejes con tracción en las 4 ruedas y suspensión para amortiguar el rebote al moverse por terreno pedregoso, [3] tal como se muestra en la figura 1.

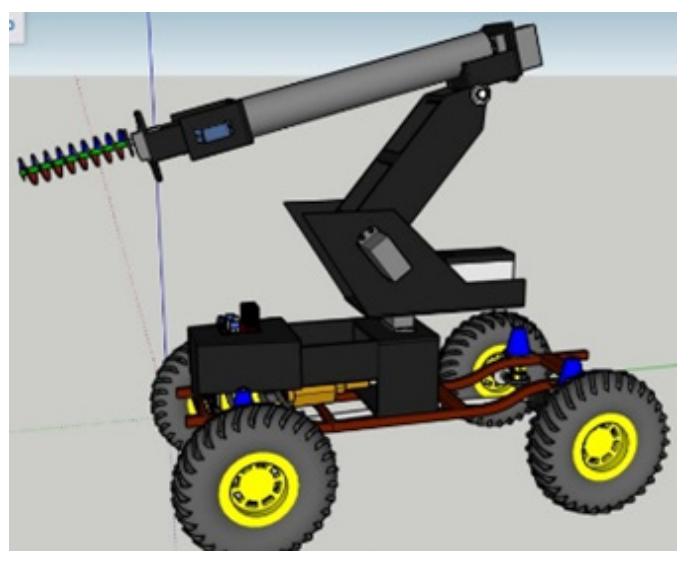

Figura 1. Estructura del robot. Elaboración propia.

\subsection{Diseño Eléctrico - Electrónico}

Se presenta los componentes eléctrico-electrónicos del robot diseñado, tablas de consumo de energía y esquema eléctrico:

- Arduino Mega, tarjeta de desarrollo que utiliza el microcontrolador ATmega2560. Posee 54 entradas/salidas digitales (15 pueden usarse como PWM), 16 entradas analógicas, 4 UARTs (puertos seriales por hardware).

- Servomotor $15 \mathrm{KG}, 1.5 \mathrm{KG}, 8 \mathrm{KG}, 4 \mathrm{KG}$, con enconder.

- Convertidor de voltaje LM 2596. Soporta corrientes de salida de hasta 3A, voltaje de entrada entre $4.5 \mathrm{~V}$ a $40 \mathrm{~V}$ y voltaje de salida entre $1.23 \mathrm{~V}$ a $37 \mathrm{~V}$. El voltaje de salida se selecciona mediante un potenciómetro multivuelta.

- Módulo NRF24L01 de 2.4GHz de largo alcance. Basado en el chip nRF24L01+, incorpora además el chip MCP01, el cual conforma el amplificador de potencia de RF y el preamplificador de bajo ruido (LNA) para el receptor. 
- Sensor de temperatura y humedad DHT11. La salida suministrada es de tipo digital utilizando solamente 1 pin de datos.

- Sensor inductivo para detectar objetos metálicos.

En la tabla 1, se muestra el consumo de los componentes antes listados para el robot.

TABLA 1. Consumo de los componentes del robot diseñado

\begin{tabular}{|l|c|c|c|}
\cline { 2 - 4 } \multicolumn{1}{c|}{} & Voltaje(V) & Amperaje(A) & Cantidad \\
\hline Motores y controlador & 9 & 1 & 1 \\
\hline Servomotores & 6 & 0.5 & 5 \\
\hline Sensores & 5 & 0.5 & 3 \\
\hline Regulador de energía & 9 & 2.0 & 2 \\
\hline
\end{tabular}

Elaboración propia.

En la Figura 2, se muestra el esquema eléctrico diseñado para el robot explorador:

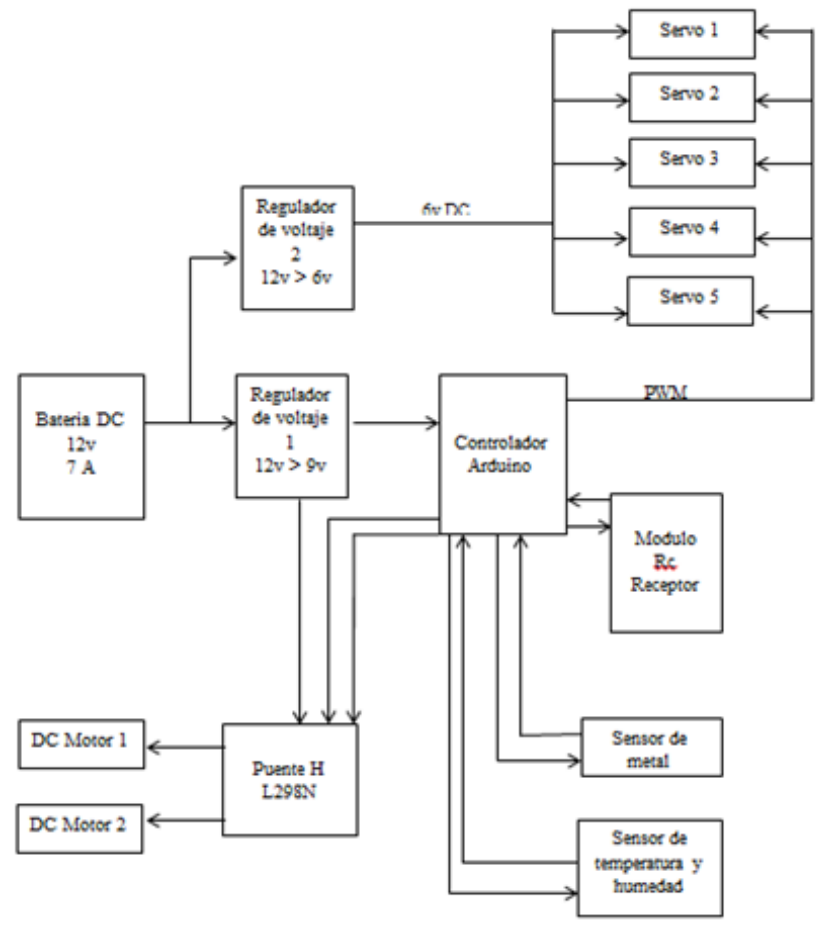

Figura 2. Esquema eléctrico del robot explorador. Elaboración propia.

\section{DISEÑO EN SOLIDWORKS}

Se procedió a diseñar la pieza para tener el origen de nuestra altura del brazo y los grados de libertad sin sufrir fallas de distribución de equilibrio [4]. En la figura 3, se muestra el diseño de la estructura base. 
MECATRÓNICA
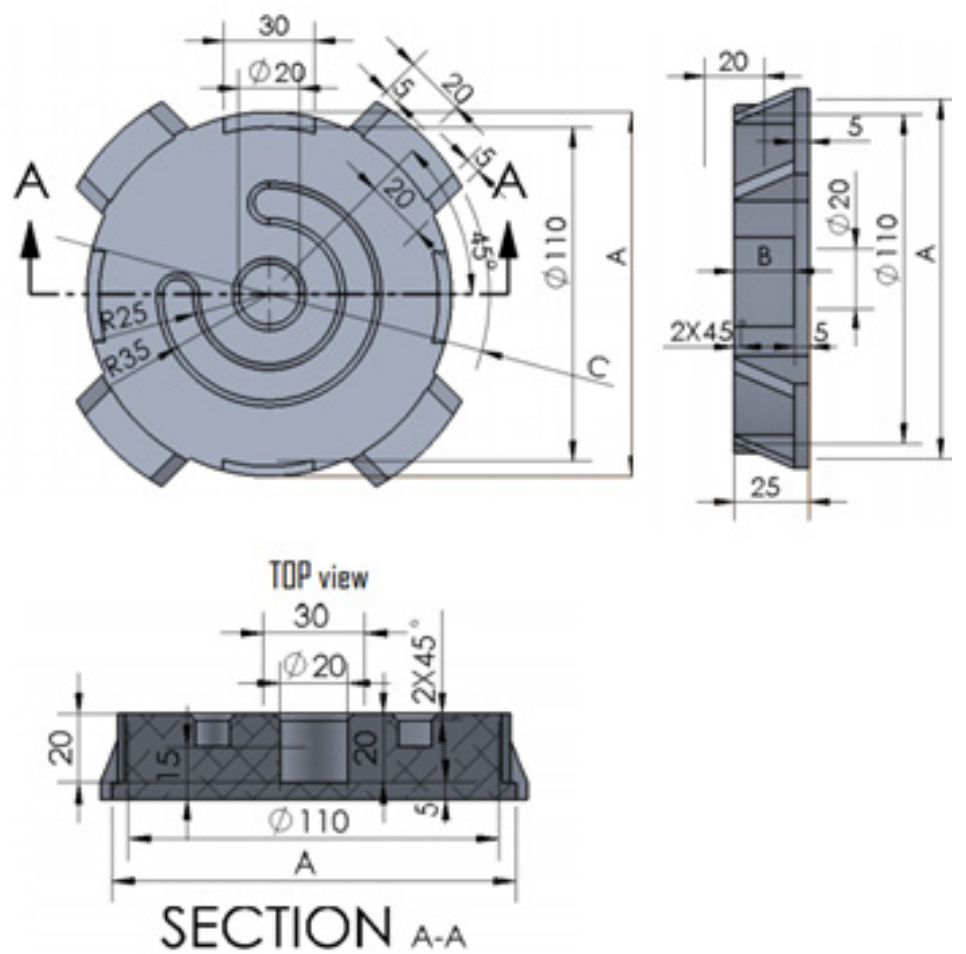

Figura 3. Estructura de la base del robot explorador. Elaboración propia.

Se diseñó la base secundaria que sujeta y permite asegurar la libertad de $360^{\circ}$, tal como se muestra en la figura 4.
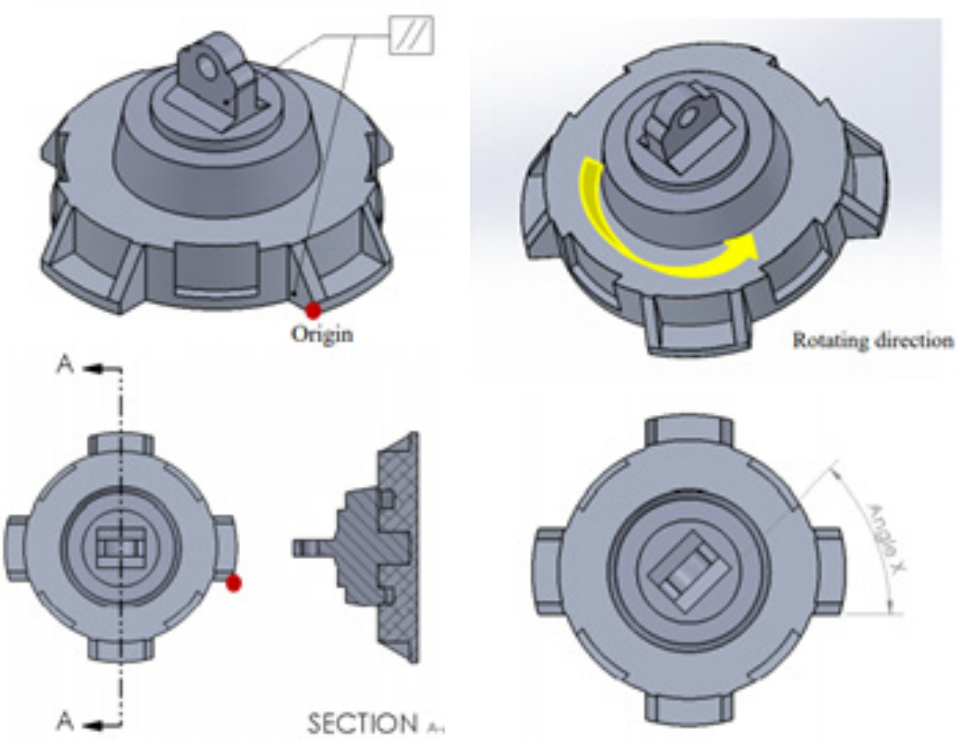

Figura 4. Estructura de la base del robot explorador. Elaboración propia. 
En la figura 5, se muestra el eslabón rígido del brazo, cuya función es la de sujetar el rotador.
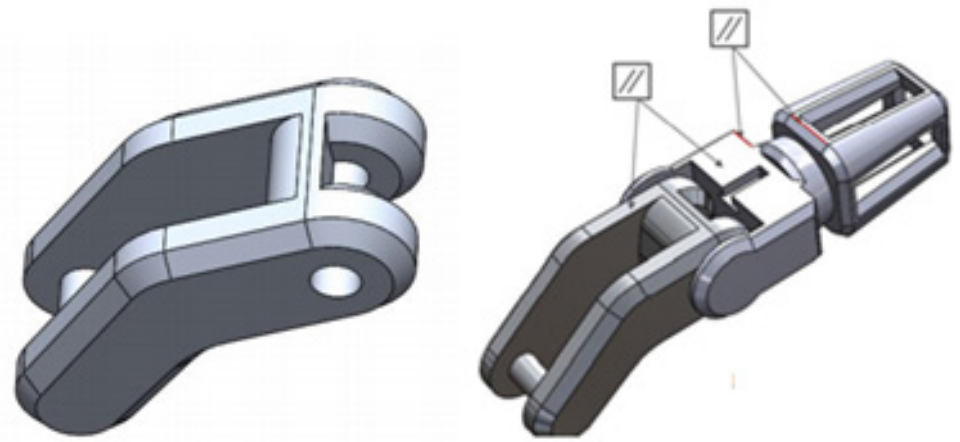

Figura 5. Estructura el eslabón rígido del brazo del robot explorador. Elaboración propia.

En la figura 6, se muestra el ensamblaje de la base con el brazo del robot explorador.
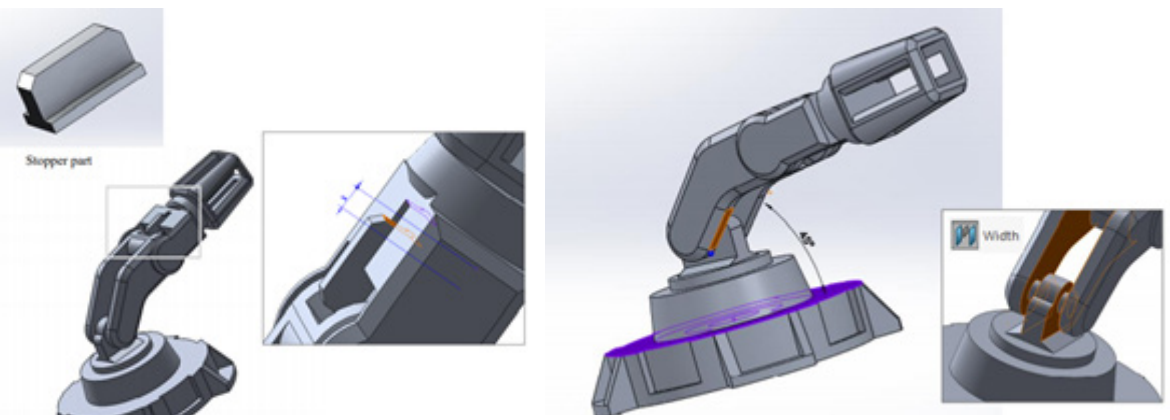

Figura 6. Ensamblaje de la base y el brazo del robot explorador. Elaboración propia.

En la figura 7, se muestra el diseño desarrollado para la pinza del robot explorador.
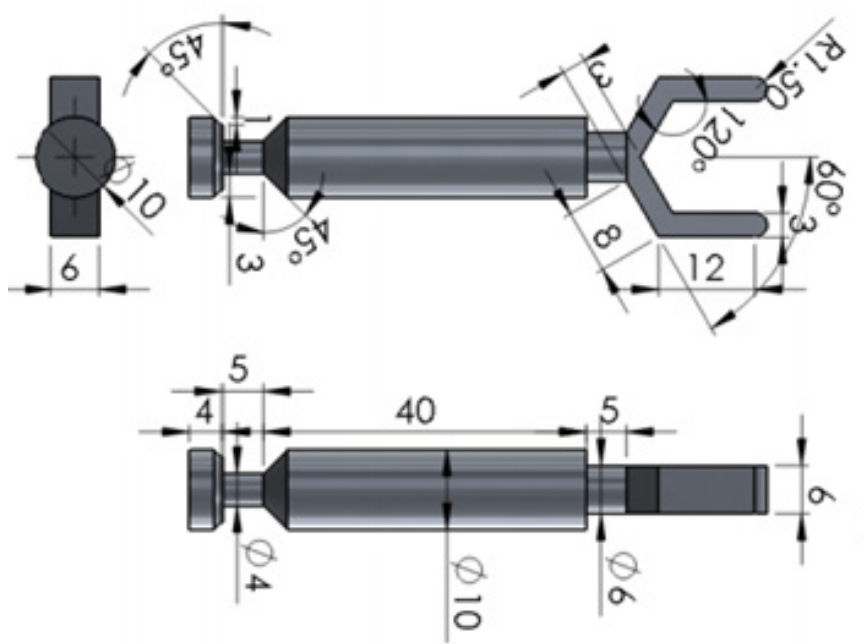

Figura 7. Diseño de la pinza del robot explorador. Elaboración propia. 
En la figura 8, se muestra el ensamblaje de la base, el brazo y la pinza del robot explorador.

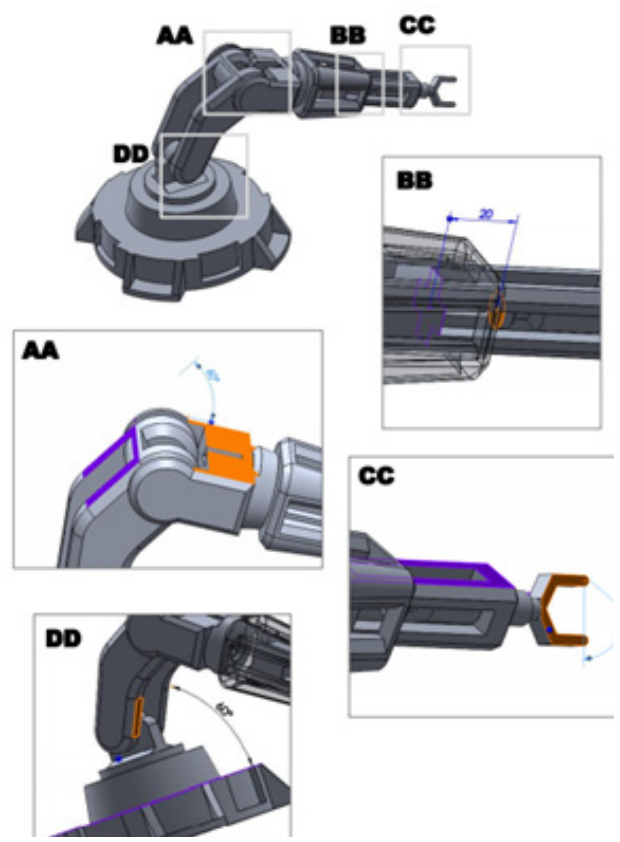

Figura 8. Ensamblaje del brazo del robot explorador. Elaboración propia.

\section{CONCLUSIONES}

Concluida la investigación se puede afirmar que se diseñó un robot explorador para la detección de metales en entornos mineros y se diseńó un prototipo digital de la estructura del brazo manipulador mediante el uso del software de ingeniería SolidWorks. Para una próxima etapa se propone realizar la implementación y pruebas que permitan demostrar el eficiente funcionamiento del robot explorador.

\section{REFERENCIAS}

[1] C. Muñoz, R. Castro, R. Palomares, yJ. Cornejo, “Mechatronics Development of Terrestrial Mobile Robot for Exploring and Monitoring Environmental Parameters at Mine Analogue Sites using IoT Platform”, 2020 IEEE XXVII International Conference on Electronics, Electrical Engineering and Computing (INTERCON), pp. 1-4 [En línea]. doi: 10.1109/INTERCON50315.2020.9220227 [Accedido: 28-dic-2020].

[2] "Boletín estadístico de la Gerencia de Supervisión Minera. Accidentes Mortales", Osinergmin, Lima, Perú, 01/2020. [En línea]. Disponible en: https://www.osinergmin.gob.pe/seccion/centro_ documental/mineria/estadisticaseindicadores/accidentes-mortales/Boletin-GSM-AccidentesMortales-2020-01.pdf [Accedido: 28-dic-2020].

[3] A. Barrientos, C. Balaguer y L. Penin, Fundamentos de Robótica, Madrid, España: McGrawHill, 2007.

[4] A. Ollero, Robótica Manipuladores y robots móviles, Barcelona, España: Marcombo, 2001. 